Human Ethology Bulletin - Proc. of the V. ISHE Summer Institute (2016): 74-83

Brief Report

\title{
CAFFEINE AND CALORIE CONSUMPTION AT A COLLEGE CAMPUS COFFEE CAFÉ
}

\author{
Jessica Sloan Kruger ${ }^{1}$, Thomas Castor ${ }^{1}$, Daniel J. Kruger ${ }^{2}$ \\ ${ }^{1}$ Health and Recreation Professions, University of Toledo, Toledo, USA. \\ ${ }^{2}$ School of Public Health, University of Michigan, Ann Arbor, Michigan, USA. \\ Jessica.Sloan2@rockets.utoledo.edu
}

\begin{abstract}
Coffee and tea consumption has substantially increased among young adults in the United States across the past few decades, particularly on college campuses. In this study, we observed beverage purchases at a campus coffee shop across an academic term at a university in the Midwestern USA. Although results do not indicate a gender difference in average drink size, women consumed more calories and a greater proportion of daily-recommended calories on average than men did. Men consumed more caffeine on average than women did. Our project demonstrates how observational research methods can provide a better understanding of health issues in a college population.
\end{abstract}

Keywords: Diet, coffee, caffeine, calories, college students.

\section{INTRODUCTION}

Coffee beverage providers are now nearly ubiquitous on North American academic campuses. These establishments serve beverages ranging from basic black coffee to specialty drinks that vary by franchise and location. Grigg (2002) found that within the past decade, coffee and other caffeinated beverages have become one of the most consumed beverage 
Sloan Kruger, J., Castor, T. \& Kruger, D.J.: Caffeine and Calorie Consumption Human Ethology Bulletin - Proc. of the V. ISHE Summer Institute (2016): 74-83

types in the world, coffee only following that of soft drinks (and plain water) in the United States. About $89 \%$ of the adult US population is a caffeine consumer on any given day. An equal percentage of men and women ingests caffeine (Fulgoni, Keast, \& Lieberman, 2015). The purpose of this study was to examine the consumption of caffeinated drinks at a university campus coffee beverage provider.

The specialty coffee drinks frequently consumed today are considerably higher in calories than the basic coffee drinks most widely available in earlier times (The Nutrition Source, 2015). Starbucks has expanded rapidly; the chain opened nearly two stores per day between 1987 and 2007, with 11,563 stores total in the USA alone at the end of 2014 (Starbucks, 2015). According to the National Coffee Association Study (2015), people aged 18-39 were more likely then older consumers to purchase coffee out-of-home. Coffee drinks range from 5 to over 500 calories per beverage (Starbucks, 2015). A previous study found that college age women consumed an additional $186 \mathrm{kcal}$ on days when they drank gourmet coffee beverages (GCB) (mean=2,017 \pm 588 ; GCBs contributed $14 \%$ of energy and $12 \%$ of energy from fat to daily intake) than on non-GCB days (mean $=1,831 \pm 530$; $p=.318$ ) (Shields, Corrales, \& Metallinos-Katsaras, 2004). The excess calories consumed in coffee beverages can increase the likelihood of obesity and other health issues (Lustig, Schmidt, \& Brindis, 2012). Thus, coffee consumption is now an important aspect of students' dietary behaviors.

Many coffee beverages are also high in caffeine. For example, the average 16 ounce Starbucks coffee contains 320 milligrams of caffeine, which is roughly 10 times that of a 12 ounce can of Coca-Cola (34 mg of caffeine) (Coca-Cola Ingredients and Nutritional Information, 2014; Starbucks, 2015a). The average cup of coffee ( 8 fluid ounces or 237 milliliters) contains 95-200mg of caffeine (Mayo Clinic Staff, 2014a). Consuming more than 500 milligrams of caffeine a day can increase the likelihood of insomnia, nervousness, restlessness, irritability, stomach upset, tachycardia, and muscle tremors (Mayo Clinic Staff, $2014 \mathrm{~b}$ ). Overconsumption of calories and caffeine can increase the likelihood of serious health consequences.

A recent study (2014) focusing on caffeine beverage intakes in the U.S. shows that caffeine intakes are slightly higher than what they were a decade ago. This study utilized a survey and diary of the beverages that people consumed and found that coffee is the largest contributor of beverage caffeine intake (Mitchell, Knight, Hockenberry, Teplansky, \& Hartman, 2014). The study found that the mean daily caffeine intake from all beverages was $165 \pm 1 \mathrm{mg}$ for all ages combined. Caffeine intake was highest for people ages 50-64 years old (226 $\pm 2 \mathrm{mg} /$ day) (Mitchell et al., 2014).

This study uses an observational approach to document and analyze the caffeine and calorie consumption of consumers at a campus coffee shop at a university in the Midwestern USA. Our purpose is to provide useful information using observational methods that will complement data from typical methods of research on health behaviors, such as self-report surveys. Survey results about food consumption are prone to recall bias, especially regarding complicated items such as specialty coffee drinks. Store records on sales of coffee beverages 
are considered to be proprietary information, and thus are nearly impossible for researchers to obtain. The observational approach to record consumer-purchasing behavior has the added benefit of documenting consumer characteristics and additional relevant behaviors (e.g., using additives such as cream and sugar). We also explored if there were sex differences in consumption patterns.

\section{METHODS}

The University of Toledo's Institutional Review Board approved the study prior to the start of observations. Observations $(N=912$ orders) were conducted at a Starbucks shop located within a student union at a university in the Midwestern USA. We conducted fifteen observation sessions, one-hour in duration, from 27 January 2015 to 5 May 2015. Observation sessions varied by time of the day and day of the week. During these observation sessions, the observers sat at a table that was approximately three meters from the counter where drink orders were announced by coffee shop employees and retrieved by the customer (See Figure 1). The observers had a clear view of the table where customers used additives such as cream and sugar. Orders were recorded when the observer heard the full order and observed the quantity of additives (e.g., three packets of sugar) added by the customer. If the order was unclear or not fully heard it was omitted from the data to ensure accuracy of the orders.

Customer gender was recorded when the customer picked up the beverage. If a customer picked up more than one beverage the beverage order was not recorded, because the gender of the consumer could not be determined. This situation occurred only 5 times during the observation period. Two observers were present in $65 \%$ of sessions to verify accuracy of orders. The reviewers verified accuracy of orders by recording orders separately on a tracking sheet and then confirming with the other observer immediately following each observed purchase. A second check of the consistency between observers was completed at the time the information was entered into the statistical software. Observation logs were compared to ensure consistency and accuracy of the information. If the observers did not agree on the beverage the entry was not recorded. Observers believed they were able to determine and reached agreement on the gender of every subject.

In accordance with Starbucks protocol, each order is verbally announced upon completion including whether there are any staff-provided additives (such as flavoring and whipped cream) or modifications to the standard recipe (extra syrup, double shots, and/or milk preference). Observers also documented whether customers added any additional cream or sugar, as well as customer gender. Nutritional information for each order was calculated based on information provided on the Starbucks website (Starbucks, 2015b). The Starbucks website has a comprehensive calorie calculator to accommodate their various ingredients, such as types of milk, syrup, whipped cream and other additives. Total calories were computed from the beverage's listed information and listed calories in beverage additives. The caffeine content of beverages is approximate. 
Sloan Kruger, J., Castor, T. \& Kruger, D.J.: Caffeine and Calorie Consumption Human Ethology Bulletin - Proc. of the V. ISHE Summer Institute (2016): 74-83

Table 1. Examples of beverage calorie and caffeine amounts.

\begin{tabular}{lcc}
\hline \multicolumn{1}{c}{ Beverage } & Caffeine & Calories \\
\hline S'mores Frappuccino & 75 & 500 \\
Java Chip Frappuccino & 110 & 470 \\
Hot Chocolate & 25 & 400 \\
Carmel Macchiato & 150 & 240 \\
Caffé Americano & 225 & 15 \\
Coffee & 330 & 5 \\
\hline
\end{tabular}

Note: Values above are based on Grande sizes (16 fluid oz/473 mL), and specialty beverages being made with $2 \%$ milk and including whipped cream.

We compared women and men's average drink size (in fluid ounces), calorie intake (in Calories), and caffeine intake (in $\mathrm{mg}$ ) with independent-samples $t$-tests for continuous measures. We computed $95 \%$ confidence intervals for aggregate scores and effect sizes for gender differences. We converted observation dates into a continuous variable indicating the number of days since the beginning of the study and examined trends in calorie and caffeine consumption over time, separately for men and women with Pearson correlations.

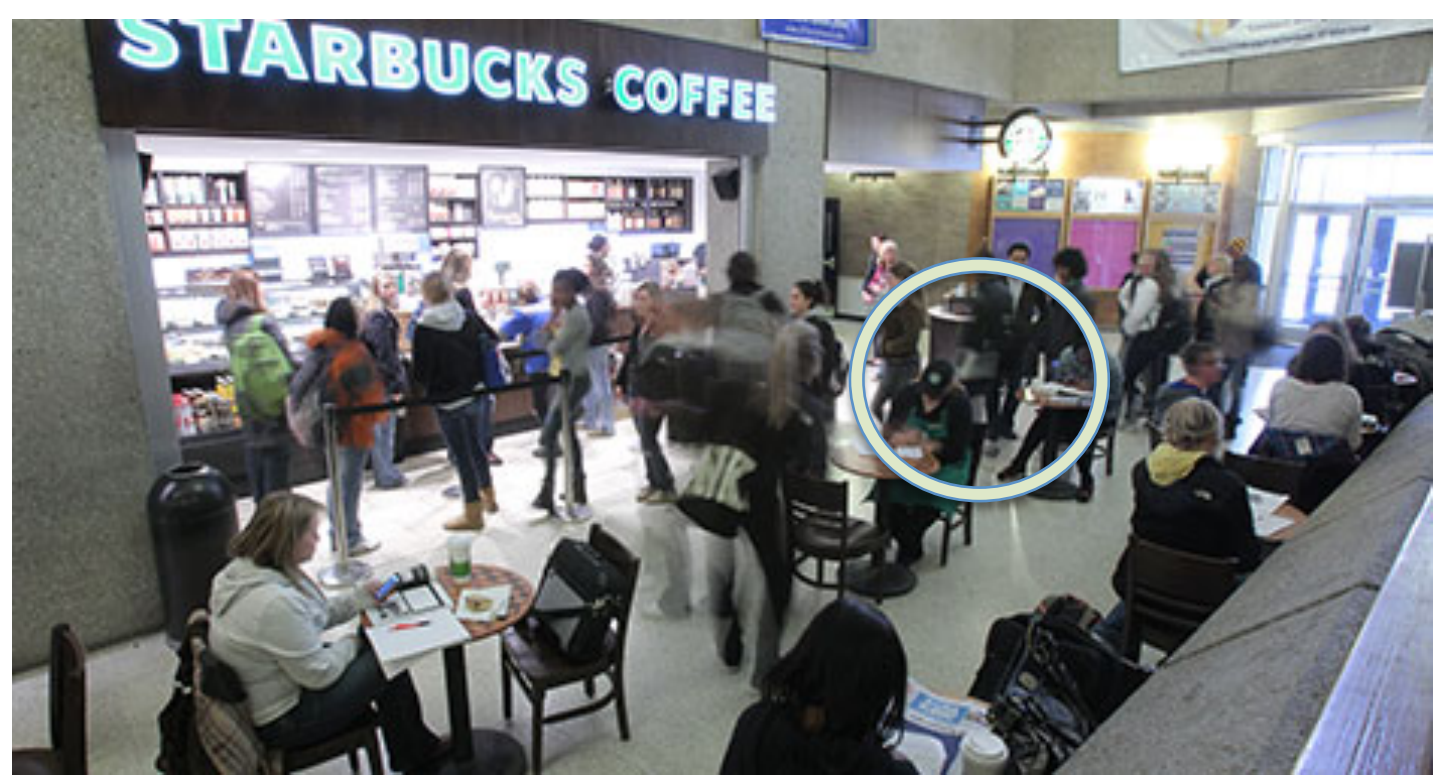

Figure 1. Field site for observations (Note: Observers were stationed three meters from the pick up location of the coffee, at the table circled on the right side in this photograph.) 


\section{RESULTS}

Women were the majority (69\%) of customers at the Starbucks. Women and men did not differ in average drink size (16 fluid ounces), $t(910)=1.10, p=.271, d=.08$ (See Figure 2 and 3). Men were more likely than women to add extra sugar ( $15 \%$ vs $4 \%, t(910)=5.92, p<$ $.001, d=.40)$, and cream ( $14 \%$ vs. $4 \%, t(910)=6.06, p<.001, d=.40)$ to their drinks. Women consumed more calories on average than men did, accounting for added sugar or creamer, 253 vs. $224, t(910)=2.56, p=.011, d=.18$ (See Figure 4 ). Men consumed more caffeine on average than women did, $169 \mathrm{mg}$ vs. $117 \mathrm{mg}, t(910)=6.02, p<.001, d=.41$ (See Figure 4). Across observations, from mid-Winter to late Spring 2015, average caffeine consumption declined for both men, $r(279)=-.166, p=.005$, and women, $r(633)=-.140, p$ $<.001$ (See Figure 5). The average calorie intake increased for men, $r(279)=.164, p=.006$, but not for women, $r(633)=.018, p=.654$ (See Figure 6).

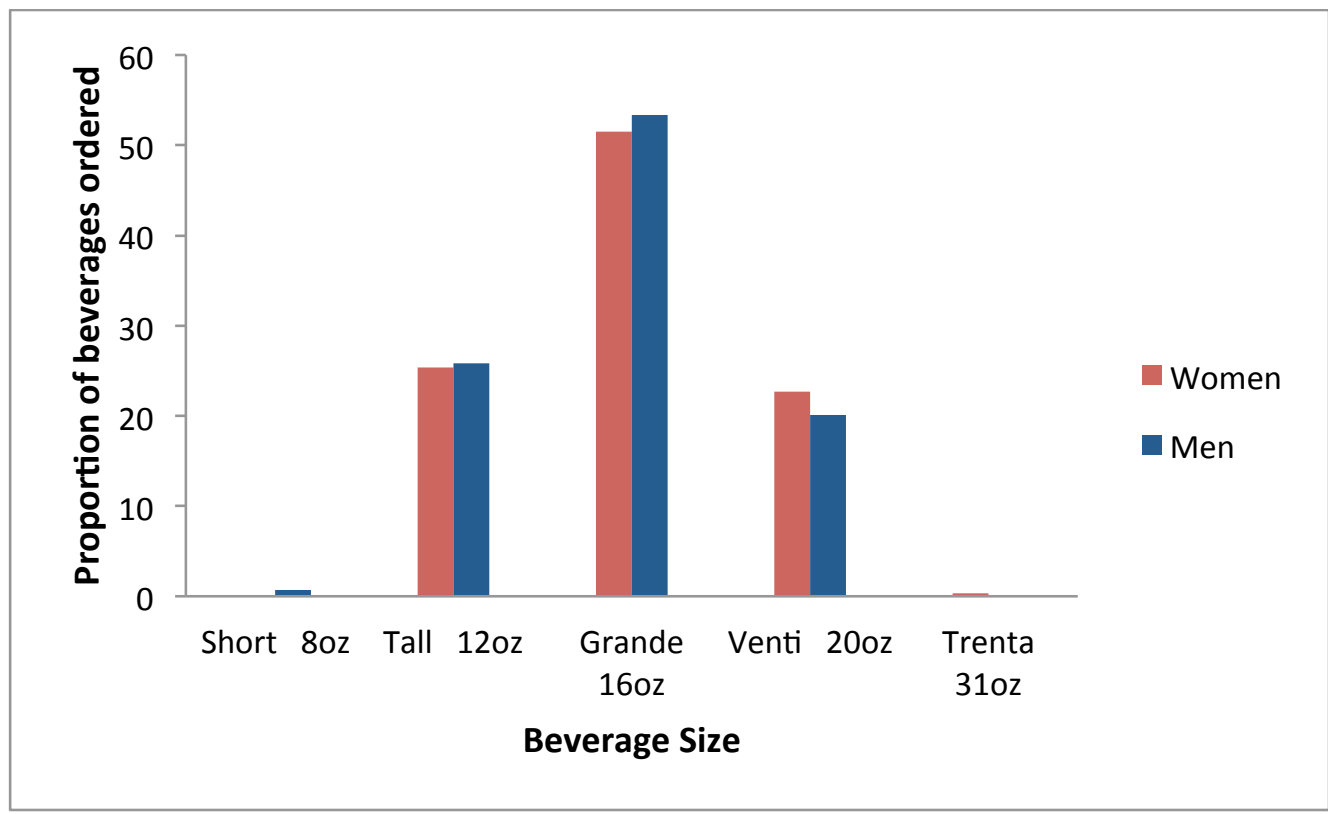

Figure 2. Proportion of Beverage Orders by Size and Customer Gender 
Sloan Kruger, J., Castor, T. \& Kruger, D.J.: Caffeine and Calorie Consumption Human Ethology Bulletin - Proc. of the V. ISHE Summer Institute (2016): 74-83

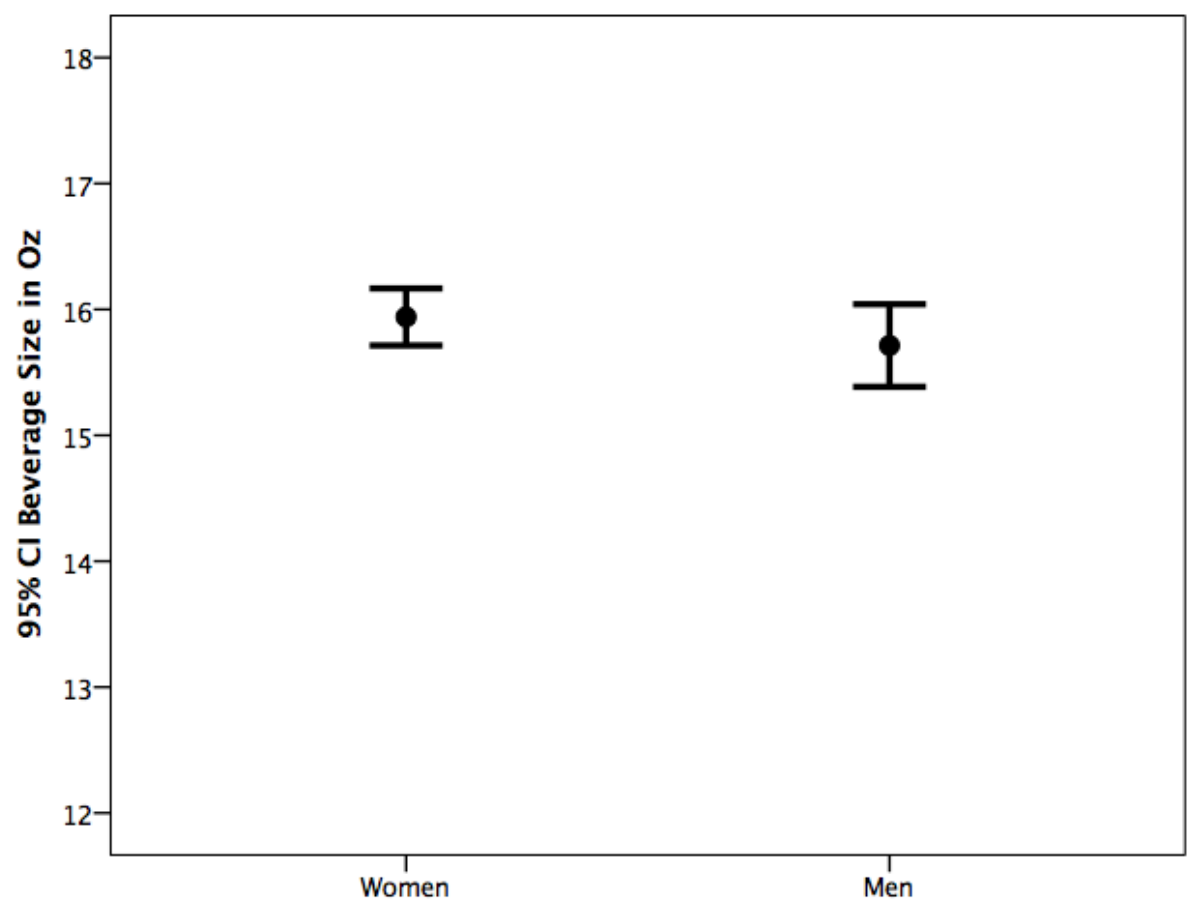

Figure 3. 95\% Confidence interval of beverage size in fluid ounces by customer gender (Note: $\mathrm{d}=$ .08 ; ds of .20, .50, and .80 respectively represent small, medium, and large effects (Cohen, 1988)).
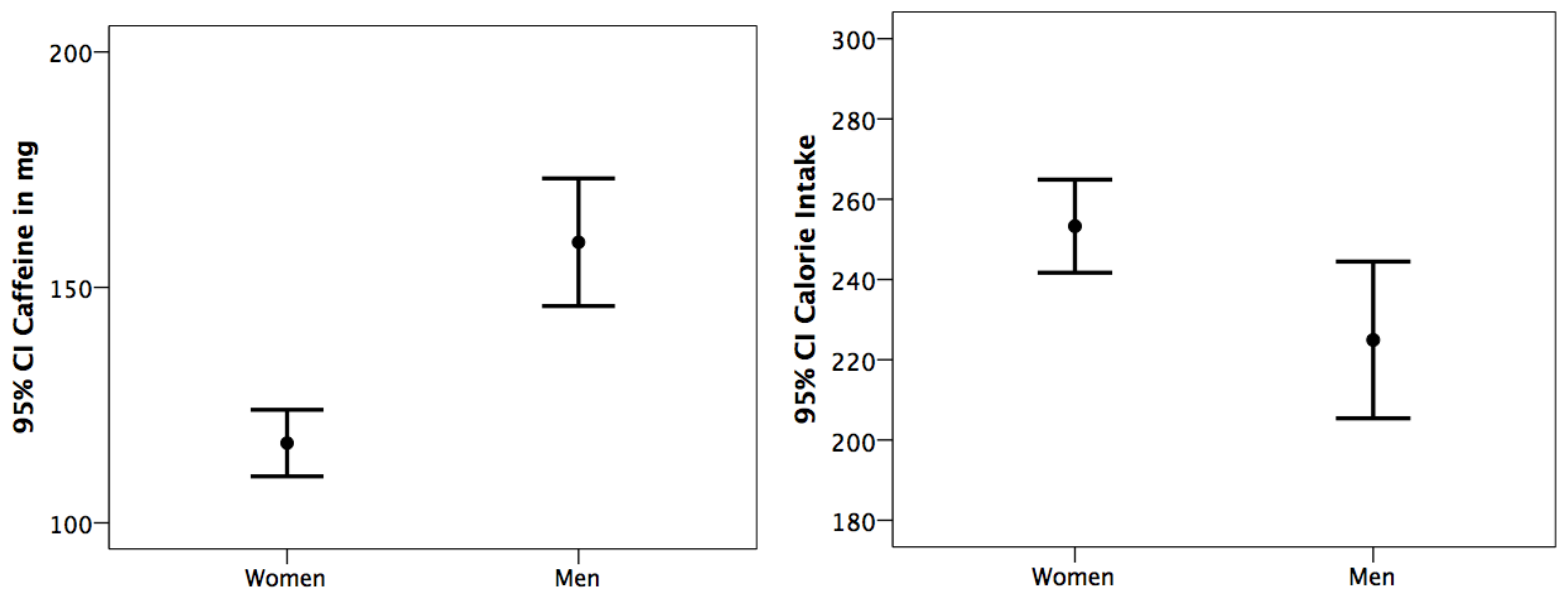

Figure 4. 95\% Confidence intervals of caffeine and calorie consumption by customer gender (Note: $\mathrm{d}=.41$ for caffeine intake, $\mathrm{d}=.18$ for calorie intake; $\mathrm{ds}$ of $.20, .50$, and .80 respectively represent small, medium, and large effects (Cohen, 1988)). 
Sloan Kruger, J., Castor, T. \& Kruger, D.J.: Caffeine and Calorie Consumption Human Ethology Bulletin - Proc. of the V. ISHE Summer Institute (2016): 74-83

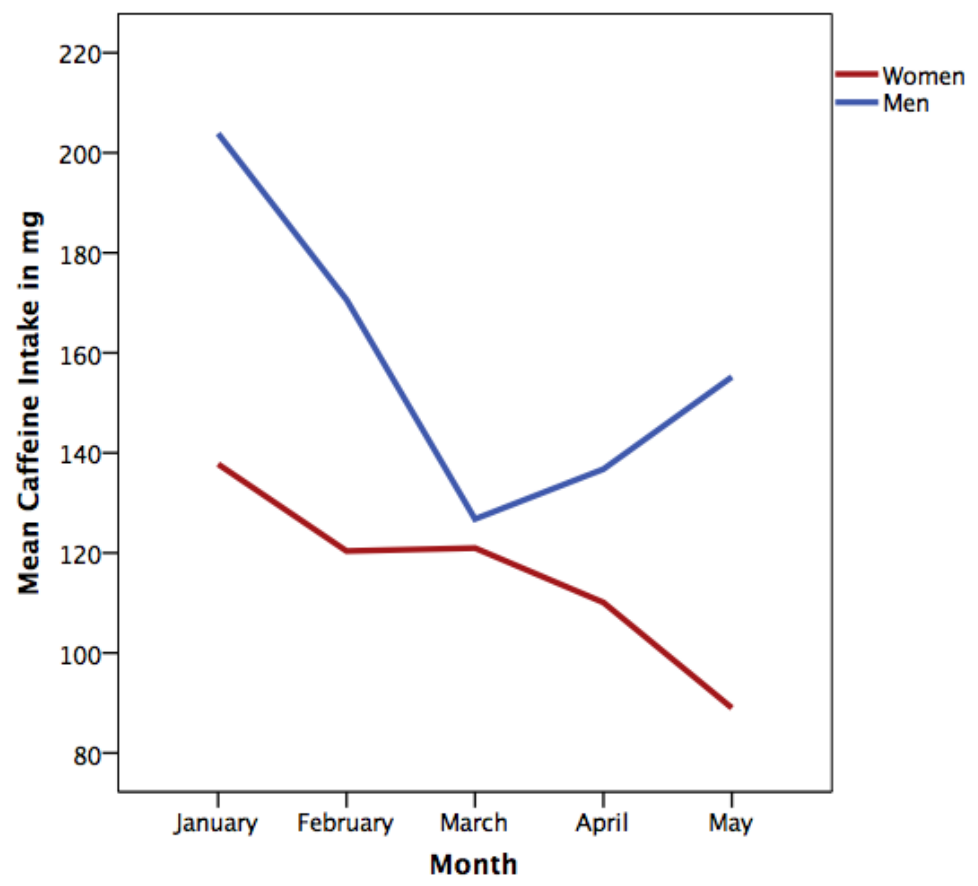

Figure 5. Mean caffeine intake by month

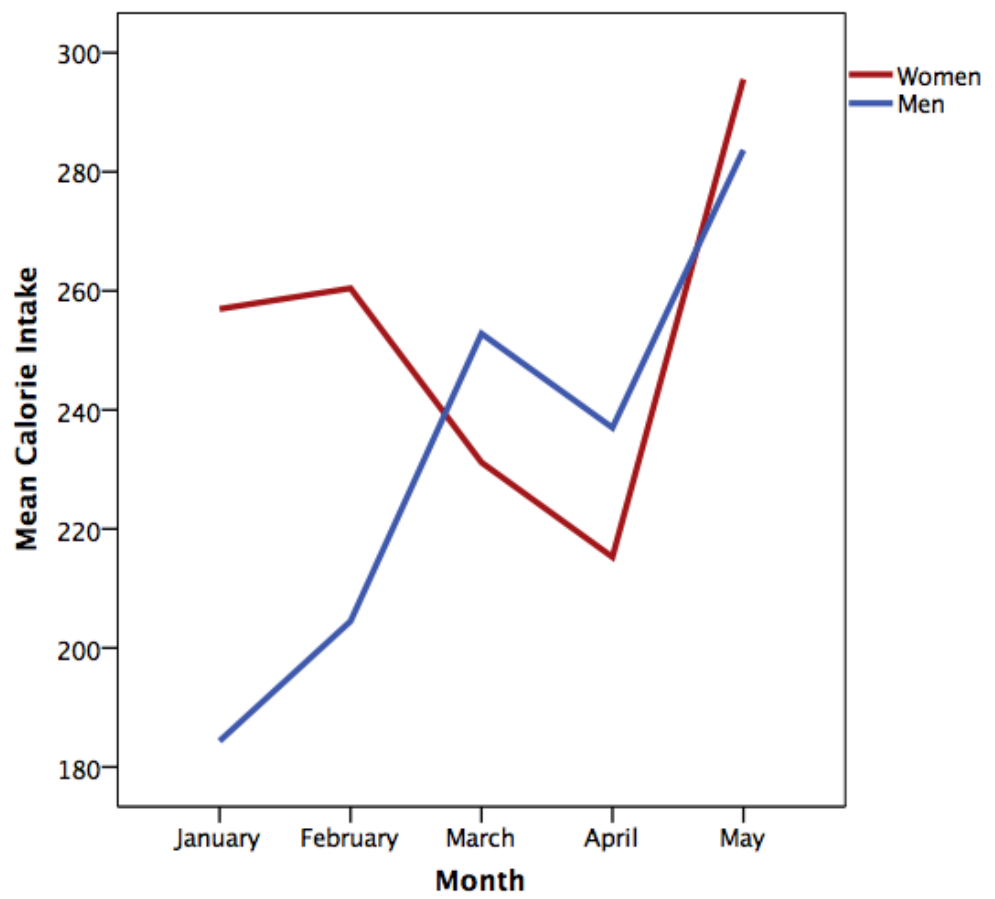

Figure 6. Mean calorie intake by month 
Sloan Kruger, J., Castor, T. \& Kruger, D.J.: Caffeine and Calorie Consumption

Human Ethology Bulletin - Proc. of the V. ISHE Summer Institute (2016): 74-83

\section{DISCUSSION}

Several studies have investigated caffeine consumption from energy drinks among college students (Attila \& Çakir, 2011; Dillon et al.; Norton, Lazev, \& Sullivan, 2011), but to our knowledge no previous study has focused on caffeine and calories from the consumption of coffee beverages. We found that women consumed a greater proportion of their recommended daily energy intake from coffee beverages than did men, $11.5 \%$ vs. $7.8 \%$, based on the US Department of Health and Human Services estimated calorie needs for moderately active women and men ages 19-25 (the age range for most undergraduates at the university). In contrast, men consumed $33.8 \%$ of the recommended daily caffeine intake, compared to $23.4 \%$ for women. Previous studies have found consistent results that men consume greater quantities of caffeine than do women (Fulgoni, Keast, \& Lieberman, 2015). Our results are also consistent with another study which found that male doctors bought significantly more coffees per person per year (128 (SD 140) v 86 (SD 86), $t=-4.66$, $\mathrm{P}<0.01$ ) and twice as many espressos as female doctors (mean 27 (SD 46) v 10 (SD 19), $\mathrm{t}=-6.54, \mathrm{P}<0.01$ ) (Giesinger, Hamilton, Erschbamer, Jost \& Giesinger, 2015). The proportions in our study are based on a single purchase, so individuals may have ingested additional calories and caffeine at other times of the day.

Mitchell et al. (2014) examined overall caffeine consumption across age groups and calculated the average amount of caffeine consumed per day, including caffeine specifically consumed in coffee beverages. For 18-24 year olds (the average age of most college students) consumption averaged $60.1 \pm 3.6 \mathrm{mg} /$ day of caffeine (Mitchell et al., 2014). Our study found that people on a university campus were consuming twice this average daily amount of caffeine from one beverage, on average men consumed 169mg of caffeine per drink and women $117 \mathrm{mg}$ of caffeine per drink. This could represent a difference between the college students we observed and the Beverage Consumption Panel, utilize by Mitchell et al. (2014), which is a sample tailored to match U.S. Census demographic characteristics, or a difference based on methodologies, i.e. self-report vs. observational. Although selfreport surveys can assess multiple beverage purchases in one day, in contrast to our observation of one purchase, the lower self-reported caffeine consumption suggests that selfreported caffeine intake might not be accurate. The self-reported data relied on caffeine values in a list of general beverages; "coffee (e.g., specialty coffee drinks, iced coffee, brewed, instant, and decaffeinated coffee), tea (e.g., green tea, white tea and other varieties, iced tea)," and "most food and nutrient databases have limited brand specificity, particularly for coffee and tea," (Mitchell et al., 2014, p. 137). Over the course of the semester, women consumed less caffeine on average. Men's caffeine consumption dropped from January to March and then increased from March to May. These trends may in part be an artifact of seasonal differences in beverage availability and gender differences in preferences. Subjects are also experiencing more intense and longer periods of sunlight as the semester progresses, and thus may require less caffeine to stay alert. Additional evidence for the causal effect of the amount of sunlight would be generated if the reverse pattern were found during the Fall semester. 
College students are also under pressure to be successful in their course work. Many feel that coffee is the "magic elixir" to yield high levels of productivity. Yet, few know the dangers of consuming too much caffeine per day. Interventions are needed to raise awareness regarding the high number of calories that coffee beverages may contain and/or the dangers of consuming too much caffeine. College students are especially vulnerable to obesity due to over consumption of calories, especially liquid calories. A media campaign could focus on increasing awareness about the number of calories in a coffee beverage and emphasize the need to observe the existing calorie counts. Along with increasing knowledge about simple ways to reduce calories, such as not adding whipped cream, selecting non-fat milk, and not adding additional syrups to beverages.

\section{LIMITATIONS}

As with any study, there are limitations. We only used one Starbucks location, on one campus in the Midwestern USA. It is unknown if people had more than one Starbucks beverage per day or other drinks with caffeine or calories. Our current research utilizes a combination of methodologies to address these limitations. The observations were also completed during the time that "holiday" beverages are available which could have possibly skewed the data. Furthermore, people are more likely to drink hot beverages when it is cold out, since the observations were completed during the winter months this could have inflated the number of beverages ordered. We used officially listed values for calorie content in coffee additives and were not able to control for individual variation in portion size.

\section{CONCLUSION}

Overall, we provided information on patterns of coffee beverage calorie and caffeine consumption in a university campus population. Our observational methods complement results from self-report survey studies. A combination of observational and survey methods may extend understanding of these issues even further.

\section{REFERENCES}

Attila, S., \& Çakir, B. (2011). Energy-drink consumption in college students and associated factors. Nutrition, 27(3), 316-322. DOI

Coca-Cola Ingredients and Nutritional Information. (2014). Retrieved from http://www.cocacolaproductfacts.com/en/coca-cola-products/coca-cola/

Cohen, J. (1988). Statistical power analysis for the behavioral sciences (2nd ed.). New York: Academic Press. DOI

Department of Health and Human Services and U.S. Department of Agriculture: Dietary Guidelines for Americans. Washington DC: HHS and USDA; 2005. 
Sloan Kruger, J., Castor, T. \& Kruger, D.J.: Caffeine and Calorie Consumption Human Ethology Bulletin - Proc. of the V. ISHE Summer Institute (2016): 74-83

Dillon, P., Svikis, D., Pomm, D., Thacker, L., Kendler, K., \& Dick, D. Understanding the buzz about energy drink use in college students. Drug \& Alcohol Dependence, 146, e267. DOI

Fulgoni, V. L., Keast, D. R., \& Lieberman, H. R. (2015). Trends in intake and sources of caffeine in the diets of US adults: 2001-2010. The American journal of clinical nutrition, 101(5), 10811087. DOI

Giesinger, K., Hamilton, D. F., Erschbamer, M., Jost, B., \& Giesinger, J. M. (2015). Black medicine: an observational study of doctors' coffee purchasing patterns at work. BMJ (Clinical Research $E d$.), 351, h6446. DOI

Grigg, D. (2002). The worlds of tea and coffee: Patterns of consumption. GeoJournal, 57, 283-294. DOI

Lustig, R. H., Schmidt, L. A., \& Brindis, C. D. (2012). Public health: The toxic truth about sugar. Nature, 27-29. DOI

Mayo Clinic Staff. (2014a, May 13). Caffeine content for coffee, tea, soda and more. Retrieved from http://www.mayoclinic.org/healthy-lifestyle/nutrition-and-healthy-eating/in-

depth/caffeine/art-20049372

Mayo Clinic Staff. (2014b, April 14). Caffeine: How much is too much? Retrieved from http://www.mayoclinic.org/healthy-lifestyle/nutrition-and-healthy-eating/indepth/caffeine/art-20045678

McIlvain, G. E., Noland, M. P., \& Bickel, R. (2011). Caffeine consumption patterns and beliefs of college freshman. American Journal of Health Education, 42, 235-244. DOI

Mitchell, D. C., Knight, C. A., Hockenberry, J., Teplansky, R., \& Hartman, T. J. (2014). Beverage caffeine intakes in the U.S. Food and Chemical Toxicology, 63, 136-142. DOI

National Coffee Association. (2015). National coffee drinking trends 2005. New York. http://www.ncausa.org/

Norton, T. R., Lazev, A. B., \& Sullivan, M. J. (2011). The "buzz" on caffeine: Patterns of caffeine use in a convenience sample of college students. Journal of Caffeine Research, 1(1), 35-40. DOI

Shields, D. H., Corrales, K. M., \& Metallinos-Katsaras, E. (2004). Gourmet coffee beverage consumption among college women. Journal of the American Dietetic Association, 104(4), 650653. DOI

Starbucks. (2015a). Nutrition. Retrieved from http://www.starbucks.co.uk/blog/archive/nutrition

Starbucks. (2015b). Explore our Menu. Retrieved from http://www.starbucks.com/menu/catalog/product?drink=brewedcoffee\#view control=nutrition

The Nutrition Source. (2015, August 26). Healthy Beverage Guidelines. Retrieved from http://www.hsph.harvard.edu/nutritionsource/nutrition-news/

Wardana, G. A., Irwadi, I., \& Soetjipto, H. (2014). Effect of coffee drinks to the reaction time of 1822 years old male university students. Folia Medica Indonesiana, 50, 48-51. 